\title{
Curcumin Inhibits Polyethylene-Induced Osteolysis via Repressing NF-kB Signaling Pathway Activation
}

\author{
Senbo An Fei Han Yihe Hu Yuwei Liu Jingyi Li Long Wang \\ Orthopedics Department, Xiangya Hospital, Central South University, Changsha, China
}

\author{
Key Words \\ Curcumin • Polyethylene (PE) • Osteolysis • RANK • NFATc1
}

\begin{abstract}
Background/Aims: Aseptic loosening is a common reason for failed artificial hip replacement after total hip arthroplasty. Aseptic loosening is mostly the result of wear debris that causes osteolysis and weakens the structures that support the prosthesis. Wear debris plays a crucial role in osteolysis during the loosening process, and polyethylene (PE) particles are found as wear debris more frequently than any other type of particle. In the absence of effective therapeutic agents, osteolysis has been hard to treat. Previous studies have demonstrated that curcumin influences signalosome-associated kinases and the proteasome-ubiquitin system during osteoclastogenesis. The aims of this study were to explore the anti-osteolysis effect of curcumin and if possible to identify the signaling pathway involved in a model of PEinduced osteolysis. Methods: Differentiation of osteoclasts was induced in vitro by PE particles in RAW264.7 (monocyte/macrophage) cells and in vivo by calvarial and air pouch models of osteolysis established by PE stimulation in mice. We performed a set of TRAP staining, realtime polymerase chain reaction (PCR), and Western blot experiments to evaluate the antiosteolytic effect of curcumin by comparing specimens that were exposed and not exposed to curcumin. Results: Curcumin had a promising inhibitory effect on osteolysis induced by wear debris and suppressed the RANK/c-Fos/NFATc1 signaling pathway. Conclusion: Curcumin can prevent PE-induced osteolysis and bone loss. An inhibitory effect on the RANK/c-Fos/NFATc1 signaling pathway may explain the anti-osteolysis activity of curcumin.
\end{abstract}

(C) 2018 The Author(s)

Published by S. Karger AG, Basel

\section{Introduction}

Total hip arthroplasty (THA) is regarded as the most effective treatment for end-stage hip disease worldwide [1]. However, with the growing number of patients undergoing this surgery, there has been an unexpected increase in the number of revision procedures required for failed THA [2]. The most frequent reasons for failure of an artificial hip joint are aseptic loosening, prosthetic joint infection, recurrent dislocation, and periprosthetic

Tel. +86 13974931684, E-Mail dr_wanglong@csu.edu.cn 


\section{Cellular Physiology Cell Physiol Biochem 2018;50:1100-1112

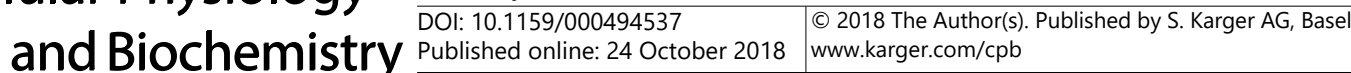 \\ An et al.: Curcumin Inhibits Osteolysis Via Repressing NF-кB}

fracture [3]. Aseptic loosening, induced mainly by wear debris formed as a result of repeated movement of an incorrectly positioned prosthesis, is the most common mechanism of failure, in which osteolysis weakens the structures that support the prosthesis [4]. Once this process starts, the quantity and quality of bone gradually deteriorates to the point that activities involving the hip become severely restricted. Wear debris plays a crucial role in osteolysis [5]. The maintenance of bone mass relies on a balance between osteogenesis by osteoblasts and bone resorption by osteoclasts [6]. Stimulation of the muscles, synovium, and articular cartilage in a joint by wear debris results in a persistent disequilibrium of bone homeostasis that leads to abnormal of osteoclasts [7]. Wear debris consists of metal, polyethylene (PE), or ceramic particles depending on the contact interface material used between the joint components. With the improvements in interface processing technology and availability of better materials [8], PE particles are found much more frequently than other particles. The osteolysis caused by these particles is hard to correct because of a lack of treatment options.

Curcumin is a yellow substance that is purified from the principal curcuminoid in turmeric (Curcuma longa) [9]. Previous studies have shown that curcumin has anti-inflammatory and chemopreventive activities. Curcumin is also involved in osteoclastogenesis via signalosome-associated kinases and the proteasome-ubiquitin system. Studies by Yang et al. [7] and Jung et al. [10] found that curcumin inhibited inflammatory activity via activation of enhancing nuclear factor erythroid-derived 2-like 2 in patients with type 2 diabetes and murine macrophages, respectively. Furthermore, curcumin is known as "the king of spices" on account of its epigenetic regulatory properties with regard to preventing cancer and neurologic and inflammatory diseases, such as breast carcinoma, osteogenic sarcoma, osteoarthritis, and neurocognitive disorders [11-14]. A recent study by Li et al. [15] showed that curcumin could suppress inflammation caused by titanium particles by regulating the polarization of macrophages. Therefore, curcumin could be developed as a treatment for inflammatory osteolysis and aseptic loosening.

Osteoclasts originate from the monocyte/macrophage lineage [16]. Previous research has confirmed that osteoblast-associated synthesis and secretion of receptor activator of nuclear factor $\kappa B$ ligand (RANKL) and osteoclast-associated RANK are essential in osteoclastogenesis and that RANK binds to RANKL on monocytes, initiating differentiation of osteoclasts [17-19]. During this process, an essential transcription factor, nuclear factor kappa B (NF- $\kappa \mathrm{B})$, is activated by the binding of RANKL and RANK via Tumor Necrosis Factor Receptor-associated Factor 6 (TRAF6) and then transported into the nucleus, where it combines with c-Fos, another transcription factor, to activate nuclear factor of activated T-cells, cytoplasmic 1 (NFATc1) to induce osteoclastogenesis [20-23]. Therefore, in this study, we used PE to induce osteoclastogenesis and explored whether c-Fos or NFATc1 was affected during this process in in vitro assays and in vivo. We also investigated whether curcumin could suppress these changes. NF- $\kappa \mathrm{B}$ is activated by phosphorylated NF- $\kappa \mathrm{B}$ inhibitor (P-IкB),

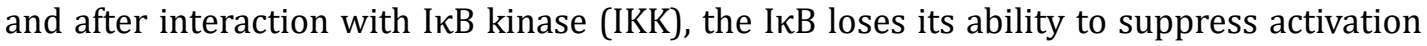
of NF- $\kappa B$. Curcumin has been shown to disable IKK, thereby preventing this process [24]. Therefore, we targeted both IкB and P-IкB to detect their roles during osteoclastogenesis. In a previous study [25], we confirmed that curcumin could reduce PE-induced osteolysis by inhibiting activation of NF- $\kappa$ B and c-Fos as well as expression of NFATc1 in a murine air pouch model. Since that study, we have continued to explore further possible mechanisms of curcumin activity. The present study could reveal a potential mechanism of anti-osteolytic activity of curcumin in the hope of identifying an innovative a therapeutic strategy for attenuating PE-induced osteolysis to some extent. 


\section{Cellular Physiology Cell Physiol Biochem 2018;50:1100-1112 \begin{tabular}{ll|l} 
and Biochemistry & $\begin{array}{l}\text { DOI: 10.1159/000494537 } \\
\text { Published onlIne: 24 October } 2018\end{array}$ & $\begin{array}{l}\text { C } 2018 \text { The Author(s). Published by S. Karger AG, Basel } \\
\text { www.karger.com/cpb }\end{array}$
\end{tabular}}

An et al.: Curcumin Inhibits Osteolysis Via Repressing NF-KB

\section{Materials and Methods}

Curcumin and differentiation of osteoclasts in RAW264.7 cells

A RAW264.7 (mouse mononuclear/macrophage) cell line was cultured in 6-well plates and fed with 2 mL of Roswell Park Memorial Institute 1640 medium (Gibco, Thermo Fisher Scientific, Inc., Waltham, MA) containing 10\% fetal calf serum (Gibco) and 1\% penicillin-streptomycin (Thermo Fisher Scientific). The cultured cell samples were allocated to treatment with phosphate-buffered saline (blank group), $1 \mathrm{mg} /$ mL PE particles (Sigma Aldrich, Inc., St Louis, MO; PE group), $1 \mathrm{mg} / \mathrm{mL}$ PE and $10 \mu \mathrm{M}$ curcumin (Sigma Aldrich; low-dose curcumin group), and $1 \mathrm{mg} / \mathrm{mL} \mathrm{PE}$ and $20 \mu \mathrm{M}$ curcumin (high-dose curcumin group). All the particles had been sterilized earlier using ethylene oxide to eliminate endotoxin after washing three times in $75 \%$ ethyl alcohol. The Limulus Amebocyte Lysate assay was used to detect endotoxin. Only

endotoxin-free particles were used in the study. PE was first suspended in culture medium and added to the samples $2 \mathrm{~h}$ after cell seeding. Curcumin was then added to the samples $0.5 \mathrm{~h}$ after addition of PE. All cells in the four treatment groups were cultured in mixed medium for $12 \mathrm{~h}$.

\section{Curcumin and differentiation of osteoclasts in mice}

Male BALB/C mice were purchased from the Experimental Animal Center at Central South University and housed in standard transparent plastic cages under standard room conditions (controlled temperature of $20 \pm 0.5^{\circ} \mathrm{C}$, a 12 -h light/dark cycle, and free access to food and water). All experimental procedures were approved by the Animal Care and Use Committee at Central South University. The study adhered to the ethical guidelines of the International Association for the Study of Pain [26]. The osteolysis model was evaluated using two methods in vivo, namely, computed tomography (CT) examination and re-creation of the environment surrounding the hip joint.

Using the first method, Briefly, 20 BALB/C mice were divided into four groups (5 mice per group). Each mouse was subjected to a surgical procedure to create a small incision in the tissues covering the skull Mice were divided into depending on the particles applied to the incisions: no PE particles (blank group), $10 \mathrm{mg}$ of PE particles (PE group), $10 \mathrm{mg}$ of PE particles in a solvent consisting of 5\% dimethyl sulfoxide (DMSO) and 95\% corn oil (solvent [negative control] group), or $10 \mathrm{mg}$ of PE particles with $10 \mathrm{mg}$ of curcumin (curcumin group). After 15 days, the mice were euthanized and the calvaria were subjected to micro-CT examination.

Using the second method, namely, re-creation of the environment surrounding the hip joint, an air pouch was created on the dorsum in 20 mice. Briefly, the hairs on the mouse's back were removed using depilatory cream. Next, 1-1.5 mL of aseptic air was injected into the subcutaneous gap on the back to form an air pouch that was maintained for a week by additional $0.5-\mathrm{mL}$ injections of air every 2 days. Skull flaps trimmed to $4 \mathrm{~mm} \times 4 \mathrm{~mm}$ in size were obtained from another 20 euthanized mice and embedded into the pre-prepared air pouches. Next, $1 \mathrm{~mL}$ of phosphate-buffered saline was injected into the flap in the air pouch in a control (blank) group ( $\mathrm{n}=5$ ) and $1 \mathrm{~mL}$ of PE suspension (mixed with normal saline $5 \mathrm{mg} / \mathrm{ml}$ ) was injected into the pre-prepared air pouch on the back of each mouse in three study groups, that is, a PE group (5 mice injected with PE only), a curcumin group ( 5 mice injected with PE followed $24 \mathrm{~h}$ later by injection of $1 \mathrm{~mL}$ of $50 \mathrm{mg} / \mathrm{kg}$ curcumin suspension), and a solvent (negative control) group (5 mice injected with 5\% DMSO and 95\% corn oil). In all groups, the experimental treatments were injected at 2-day intervals for 15 days until the mice were euthanized. The subcutaneous tissues of the air pouch were subjected to real-time PCR and Western blot analysis and cut sections of the skull flaps were used for TRAP staining.

We found that curcumin had the best solubility and the least toxicity when dissolved in 5\% DMSO and 95\% corn oil. All the surgical procedures were performed under anesthesia by intraperitoneal injection of a $10 \mathrm{mg} / \mathrm{kg}$ dose of $10 \%$ chloral hydrate (Xiangya Hospital, Central South University, Changsha, China) in a bacteria-free environment with surgical instruments that were sterilized with povidone-iodine. The mice were euthanized by dislocation of the cervical vertebrae. Tissue samples collected from these mice were flash frozen in liquid nitrogen for preservation until examination.

\section{TRAP staining}

The murine skullflaps were washed in phosphate-buffered saline, fixed with $4 \%$ paraformaldehyde for 24 $h$, and washed again in phosphate-buffered saline. Specimens were then placed in ethylenediaminetetraacetic acid decalcifying solution (provided by the Pathology Department, Xiangya Hospital) that was refreshed at 2-day intervals until the flaps could be penetrated easily by a needle (about 30-40 days). Next, the flaps were 


\section{Cellular Physiology Cell Physiol Biochem 2018;50:1100-1112 \begin{tabular}{ll|l} 
and Biochemistry Published online: 24 October 2018 & $\begin{array}{l}\text { (c) } 2018 \text { The Author(s). Published by S. Karger AG, Basel } \\
\text { www.karger.com/cpb }\end{array}$
\end{tabular}}

An et al.: Curcumin Inhibits Osteolysis Via Repressing NF-kB

dehydrated and embedded in wax. Slices with a thickness of $4 \mu \mathrm{m}$ were put on glass slides at $40^{\circ} \mathrm{C}$ and dried at $60^{\circ} \mathrm{C}$. All slices were selected from the middle area of the calvaria in all the study groups. Finally, the glass slides were subjected to TRAP staining assay (Sigma-Aldrich) according to the manufacturer's instructions. Microscopic images were captured using a DM2500 device (Leica Microsystems GmbH, Wetzlar, Germany). TRAP-positive cells were defined as cells containing $\geq 3$ nuclei/cell in 10 fields per sample at a magnification of $200 \times$.

\section{Micro-CT imaging}

The calvaria specimens were evaluated using micro-CT as described previously $[27,28]$. The calvaria were carefully excised, cleaned, and fixed immediately in $10 \%$ formalin, and then scanned with a $\mu$ CT 100 scanner (Scanco Medical AG, Brüttisellen, Switzerland). The reference lines were created from a scout view by a holder with reconstruction into $20-\mu \mathrm{m}$ voxels (volume elements) to determine the area for analysis. The microfocus X-ray tube was operated at $70 \mathrm{kV}, 200 \mu \mathrm{A}$, and $360^{\circ}$ of rotation under $300 \mathrm{~ms}$ exposure per view. Bone quality was evaluated from the bone mineral density and morphology of the skull cavity in the calvaria specimens. Tissue volume (TV), bone volume (BV), and the relative volume of calcified tissue in the selected volume of interest (BV/TV) were calculated using three-dimensional analysis software (Scanco Medical).

\section{Real-time quantitative PCR}

The total RNA in the RAW264.7 cells and the subcutaneous tissues were extracted using TRIzol (Invitrogen, Thermo Fisher Scientific) according to the manufacturer's instructions. Each $1 \mu \mathrm{g}$ of total RNA was reverse-transcribed into complementary DNA using a First Strand cDNA synthesis kit (GeneCopoeia, Rockville, MD) following the manufacturer's instructions. Quantitative PCR was performed using an Applied Biosystems 7700 Real-time PCR system (Thermo Fisher Scientific) with SYBR Green Real-time PCR Master Mix (Toyobo, Osaka, Japan) in a total reaction volume of $10 \mu \mathrm{l}$ according to the manufacturer's instructions. The thermal cycles were as follows: initial denaturation at $95^{\circ} \mathrm{C}$ for $2 \mathrm{~min} ; 45$ cycles of denaturation at $95^{\circ} \mathrm{C}$ for $15 \mathrm{~s}$; annealing at $58^{\circ} \mathrm{C}$ for $15 \mathrm{~s}$; and extension at $72^{\circ} \mathrm{C}$ for $45 \mathrm{~s}$. Relative c-Fos and NFATc 1 mRNA expression levels were calculated using the $2-\Delta \Delta \mathrm{Ct}$ method and normalized to the internal control, $\beta$-actin. c-Fos primers used were 5'-TACTACCATTCCCCAGCCGA-3' (forward) and 5'-GCTGTCACCGTGGGGATAAA-3' (reverse). NFATc1 primers used were 5'-TACTACCATTCCCCAGCCGA-3' (forward) and 5'-GCTGTCACCGTGGGGATAAA-3' (reverse). $\beta$-actin primers used were 5'-CCTGGCACCCAGCACAAT-3' \forward冈and 5'-GGGCCGGACTCGTCATAC-3' (reverse).

\section{Western blotting}

The collected RAW264.7 cells and subcutaneous tissues were homogenized and lysed in 1:100 RIPA lysis buffer (Beyotime Institute of Biotechnology, Haimen, China) containing phenylmethanesulfonyl fluoride (Beyotime Institute of Biotechnology) as per the operating instructions. A total of $30 \mu \mathrm{g}$ of lysate supernatant were separated by $10 \%$ sodium dodecyl sulfate-polyacrylamide gel electrophoresis, transferred onto polyvinylidene difluoride membranes (Beyotime Institute of Biotechnology), and blocked with $5 \%(\mathrm{w} / \mathrm{v})$ bovine serum albumin (Beyotime Institute of Biotechnology) in Tris-buffered saline with $0.1 \%(\mathrm{w} / \mathrm{v}$ ) Tween 20 . The blocked membranes were reacted with the following primary antibodies: rabbit anti-c-Fos (1:2000; Abcam Biotechnology, Cambridge, MA), rabbit anti-NFATc1 (1:500; Affinity Biosciences,

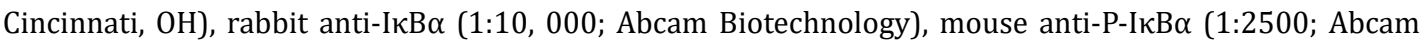
Biotechnology), mouse anti- $\beta$-actin (1:4000; Proteintech, Rosemont, IL) and rabbit anti-GAPDH (1:2000; Proteintech) at $4^{\circ} \mathrm{C}$ overnight. After four washes in Tris-buffered saline with $0.1 \%(\mathrm{w} / \mathrm{v})$ Tween 20 , the membranes were reacted with secondary antibodies conjugated to horseradish peroxidase, namely, goat anti-rabbit (1:8000) and goat anti-mouse (1:6500) IgG (both from Proteintech). The targeting proteins were visualized by reaction with an enhanced chemiluminescence system (Bio-Rad Laboratories, Hercules, CA). The target protein quantity was analyzed densitometrically using Quantity One software (version 4.0, Bio-Rad) and normalized to the corresponding $\beta$-actin or GAPDH. 


\section{Equipment and settings}

Figures 1a, 1b were captured using the DM2500 microscope at a magnification of 200x. Fig. 2A was captured by the $\mu \mathrm{CT} 100$ scanner and processed using the three-dimensional analysis software provided by Scanco Medical. Figures 4a-4d were captured with the Bio-Rad enhanced chemiluminescence system. Figures. 1c, 1d, 2b, 3, 4e, and 4f were generated by using GraphPad Prism 7 software (GraphPad Software Inc., La Jolla, CA). Figures 1-4 were combined using Photoshop CC software (Adobe, San Jose, CA). No other processing manipulations except for combination and cut were used with Photoshop. Therefore, the figures correctly represent the original data.

\section{Statistical analysis}

All the experiments were carried out in triplicate. Data were calculated using GraphPad Prism 7 software and are shown as the mean \pm standard deviation. Groups were compared using one-way analysis of variance. A P-value $<0.05$ was considered statistically significant.

\section{Results}

\section{Differentiation of osteoclasts induced by PE in vitro and in vivo}

Erythrostained multinuclear osteoclasts were identified in the PE group $(27.40 \pm 3.9$, $\mathrm{P}<0.01)$. There were significantly fewer osteoclasts in the low-dose $(17.13 \pm 4.55, \mathrm{P}<0.05)$ and high-dose $(18.1 \pm 4.13, \mathrm{P}<0.05)$ curcumin groups than in the blank group, which contained almost no osteoclasts $(0.61 \pm 1.02)$. In the skull flaps, significantly higher numbers of osteoclasts were found in the PE group $(64.72 \pm 5.53, \mathrm{P}<0.01)$, followed by the solvent $(52.86 \pm 5.2, \mathrm{P}<0.05)$ and curcumin $(31.3 \pm 8.84, \mathrm{P}<0.05)$ groups when compared with the blank group (10.81 \pm 2.96 ; Fig. 1 . On micro-CT, the volume of interest (VOI, BV/TV) was largest in the blank group $(0.73 \pm 0.02)$, followed by the curcumin group $(0.65 \pm 0.02, \mathrm{P}<0.05$ vs the blank group), the solvent group $(0.57 \pm 0.02, \mathrm{P}<0.05)$, and finally the PE group $(0.45$ \pm 0.04 ), which had the most severe bone loss. High expression of TRAP has been found in osteoclasts [29], so TRAP staining was used to identify osteoclasts in this study. In the in vitro cell experiments, the multinuclear RAW264.7 cells treated with $1 \mathrm{mg} / \mathrm{mL}$ PE stained a clear pink color by TRAP whereas the blank control group did not (Fig. 1a, magnification $200 \times$ and $400 \times$ ). Similarly, the murine calvaria specimens treated with $10 \mathrm{mg}$ of PE particles showed an abundance of pink-stained multinuclear osteoclasts and the blank control group did not (Fig. 1b, magnification 200x and 400x). The number of osteoclasts containing more than 3 pink-stained nuclei were counted in 10 random fields per sample and are compared in Fig. $1 \mathrm{c}$ and $1 \mathrm{~d}$. The number of osteoclasts in the PE group was significantly greater than that in the blank group for both the RAW264.7 cells (Fig. 1c) and murine calvaria samples (Fig. 1d, $\mathrm{P}<0.01)$. In the VOI (BV/TV) for the micro-CT assays in the murine calvarial bone samples, the bone density in the PE-treated group decreased to a greater extent than in the blank group (Fig. 2a). There was a marked increase in the bone loss index (BV/TV) in the PE group when compared with the value in the blank group (Fig. 2b). These data indicate that PE has the potential to promote differentiation of osteoclasts from monocyte/macrophages in bone.

\section{Curcumin prevents PE-induced differentiation of osteoclasts}

The osteoclast differentiation induced by PE was prevented after the RAW264.7 cells were treated with curcumin at a low dose of $10 \mathrm{M}$ or a high dose of $20 \mu \mathrm{M}$, as indicated by a decrease in the number of TRAP-stained cells (Fig. 1a, magnification 200× and 400×). Similarly, there was a marked decline in the number of PE-induced (TRAP-positive) osteoclasts in the murine calvarial bone specimens treated with $1 \mathrm{~mL}$ of curcumin $50 \mathrm{mg} / \mathrm{kg}$. Furthermore, PE-induced differentiation of osteoclasts could not be blocked in the solvent group, in which the number of multinuclear TRAP-positive cells was only slightly decreased in comparison with the PE group (Fig. 1b, magnification 200x and 400x). The digitalized osteoclast data showed that the numbers of osteoclasts in the RAW264.7cells treated with low or high doses of curcumin (Fig. 1c) and in the murine calvaria samples (the curcumin group in Fig. 1d) 


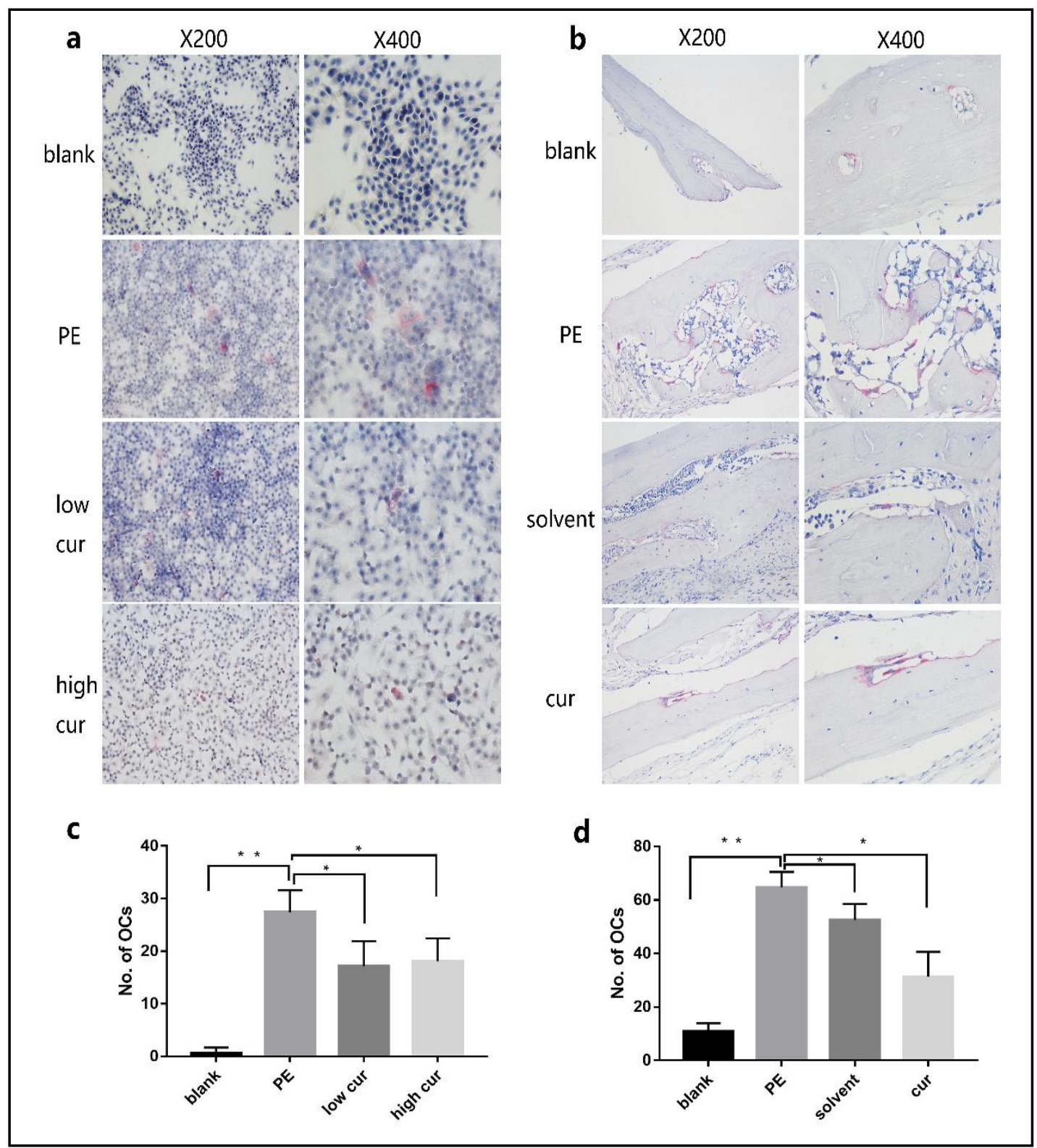

Fig. 1. Curcumin prevents PE-induced differentiation of osteoclasts. (a-d) were combined using Photoshop software. (a) Results of TRAP staining of RAW264.7 cells in the blank (non-treated) group, PE (1 mg/mL PEtreated) group, low-dose curcumin (PE plus $10 \mathrm{mM}$ curcumin-treated) group, and high-dose (PE plus $20 \mathrm{mM}$ curcumin-treated) group (left panels, magnification 200×; right panels, magnification $400 \times$ ). (b) Results of TRAP staining of murine calvaria (left panels, magnification 200x; right panels, magnification $400 \times$ ) in the blank, PE, solvent, and curcumin groups. (c) Corresponding osteoclast counts in each group of RAW264.7 cell samples. (d) The corresponding osteoclast counts in each group of calvarial bone slices. (a) and (b) are typical pictures obtained using three independent staining experiments. The data in (c) and (d) are for three independent assays and sets of results. ${ }^{*} \mathrm{P}<0.05,{ }^{* *} \mathrm{P}<0.01$. $\mathrm{PE}$, polyethylene.

were significantly lower than the number of osteoclasts in the PE group $(p<0.05)$. In the VOI (BV/TV) for the micro-CT assays in the murine calvarial bone experiment, the bone density in the curcumin-treated groups showed obvious recovery when compared with the PE group (Fig. 2a). The bone loss index (BV/TV) was markedly higher in the curcumin group than in the PE group (Fig. 2b).

\section{KARGER}




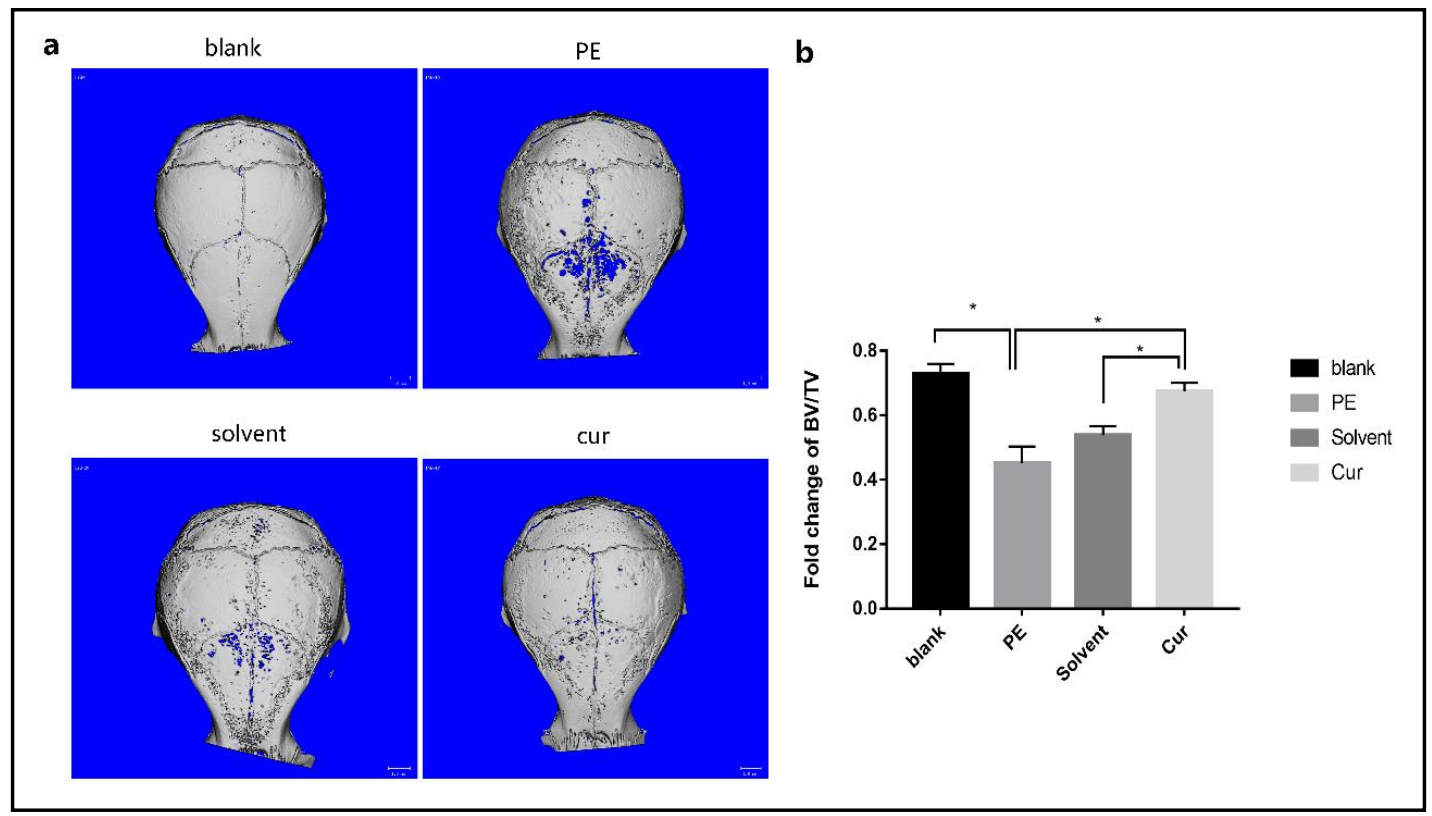

Fig. 2. Micro-computed tomography image showing that curcumin prevents PE-induced bone loss. (a) and (b) were combined using Photoshop software. (a) Typical micro-computed tomography images showing bone density in murine calvaria samples treated with PE, PE + solvent (dimethyl sulfoxide and corn oil), PE $+10 \mathrm{mg}$ of curcumin, or no treatment. (b) Corresponding bone volume/tissue volume indices. The data for each group represent at least three independent assays. ${ }^{*} \mathrm{P}<0.05$. $\mathrm{PE}$, polyethylene.

\section{a}

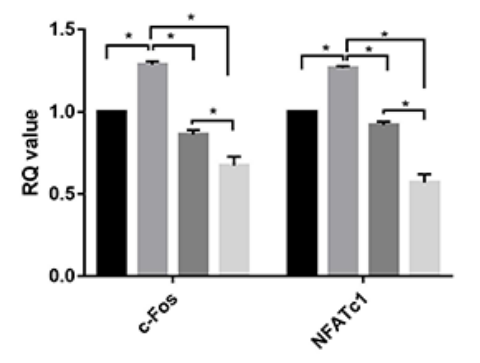

b

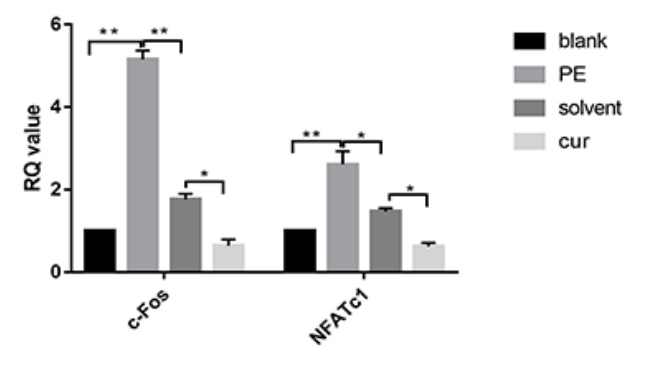

Fig. 3. Curcumin prevents PE-induced expression of c-Fos and NFATc-1 genes. (a) and (b) were combined using Photoshop software. Expression of c-Fos and NFATc1 mRNA was examined in RAW264.7 cells (a) and air pouch tissue samples (b) treated with the corresponding interventions. Non-treated (blank) samples were used as controls. Gene expression levels were normalized to the corresponding beta-actin value and are presented as folds of the blank value. ${ }^{*} \mathrm{P}<0.05,{ }^{* *} \mathrm{P}<0.01$. $\mathrm{PE}$, polyethylene.

\section{Curcumin prevents PE-triggered RANK/c-Fos and NFATc1 signaling}

Analysis of the relative quantification values showed that expression levels of c-Fos and NFATc1 increased when the RAW264.7 cells (c-Fos, $1.29 \pm 0.02, \mathrm{P}<0.05$; NFATc1, 1.27 $\pm 0.01, \mathrm{P}<0.05$ ) and air pouch tissue samples (c-Fos, $5.15 \pm 0.18, \mathrm{P}<0.01$; NFATc1, $2.61 \pm$ $0.26, \mathrm{P}<0.05$ ) were treated with $\mathrm{PE}$ particles when compared with the expression levels in the blank group (normalized as 1.00). The curcumin group showed less expression of c-Fos and NFATc1 than the PE group in vitro. The group that received curcumin $10 \mu \mathrm{M}$ showed significantly higher expression levels of c-Fos and NFATc1 $(0.86 \pm 0.02$ and $0.92 \pm$ 0.02 , respectively, both $\mathrm{P}<0.05)$ than the group that received $20 \mu \mathrm{M}$ curcumin $(0.67 \pm 0.04$ and $0.57 \pm 0.04$, respectively). In the in vivo studies, the solvent group showed significantly 


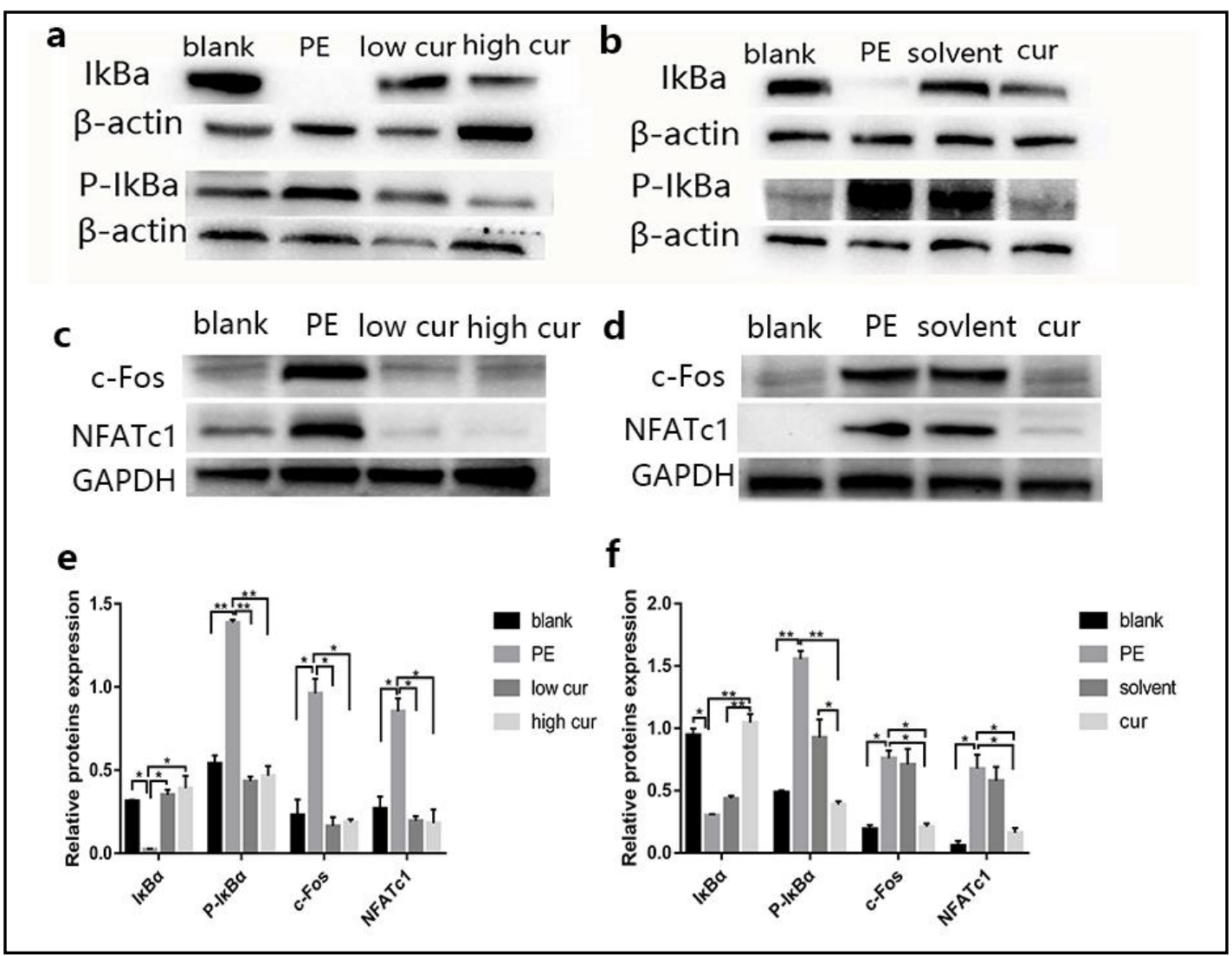

Fig. 4. Curcumin prevents a PE-induced change in the activity of I $\mathrm{B} \alpha$ protein. (a-f) were combined using Photoshop software. The blots were cropped using Photoshop and are compliant with the digital image and integrity polices of Oncotarget. c-Fos and NFATc1 protein levels as well as IkB $\alpha$ protein expression and activity (phosphorylation) were detected using Western blot in RAW264.7 cells treated with PE, low-dose curcumin, and high-dose curcumin $(a, c)$ and air pouch tissue samples (b, d) treated with PE, solvent, or PE + curcumin. Non-treated (blank) samples were used as controls. The upper panels show typical results for Western blot images. The quantity of the corresponding protein and levels of the phosphorylated protein were estimated by densitometry (lower panels). Protein expression and phosphorylation levels were normalized to the corresponding beta-actin or GAPDH value and are presented as the folds of the blank. Data for each group represent at least three independent assays. ${ }^{*} \mathrm{P}<0.05,{ }^{*} \mathrm{P}<0.01$. $\mathrm{PE}$, polyethylene.

higher expression levels of c-Fos and NFATc1 $(1.76 \pm 0.11$ and $1.47 \pm 0.07$, respectively, both $\mathrm{P}<0.05)$ than the curcumin group $(0.64 \pm 0.12$ and $0.63 \pm 0.07$, respectively). RANK, c-Fos, and NFATc1 have been reported to be involved in differentiation of osteoclasts; therefore, we analyzed the expression of their genes during PE-induced differentiation of osteoclasts with and without curcumin by quantitative PCR or Western blot in RAW264.7 cells and the air pouch tissue samples. Quantitative PCR results for the cell samples highlighted a similar tendency for c-Fos and NFATc1 mRNA expression. Expression of these genes was higher in the PE group than in the control (blank) group ( $\mathrm{P}<0.05$; Fig. 3a). However, levels of expression of the genes were significantly downregulated in the low-dose and high-dose curcumin groups $(\mathrm{P}<0.05)$ when compared with the PE group. In addition, the high-dose $(20 \mu \mathrm{M})$ curcumin group showed more efficient inhibition of both genes than the low-dose $(10 \mu \mathrm{M})$ curcumin group $(\mathrm{P}<0.05)$. In the air pouch tissue samples, expression of $\mathrm{c}$-Fos and NFATc1 mRNA demonstrated a similar tendency to that in the PE group, namely, higher expression levels than the corresponding controls (blank group, $\mathrm{P}<0.01$; Fig. $3 \mathrm{~b}$ ). c-Fos and NFATc-1 gene expression levels were significantly higher in the solvent group than in the control group (both $\mathrm{P}<0.01$ ) and were also higher than in the PE group $(\mathrm{P}<0.05)$. These

\section{KARGER}




\section{Cellular Physiology Cell Physiol Biochem 2018;50:1100-1112 and Biochemistry \begin{tabular}{l|l} 
DOI: 10.1159/000494537 & $\begin{array}{l}\text { C } 2018 \text { The Author(s). Published by S. Karger AG, Basel } \\
\text { www.karger.com/cpb }\end{array}$
\end{tabular}

Fig. 5. The mechanism schematic of curcumin inhibiting osteoclastogenesis via $\mathrm{NF}-\kappa \mathrm{B}$ signaling pathway. Initially, excessive RANKL combines with the trans-membrane receptor RANK in osteoclast precursors, activating IKK through TRAF6, then IKK deactivates I $\kappa$ B through phosphorylation so that P-I $\mathrm{B}$ separates from NF- $\mathrm{B}$ and loses the ability to suppress NF- $\kappa$ B. Consquently, NF- $\kappa$ B is activated and transported into the nucleus, where it combines with the key transcription factors, c-Fos and NFATc1 to induce osteoclastogenesis. Curcumin can disable IKK so that it can prevent the consequent osteoclastogenesis process.

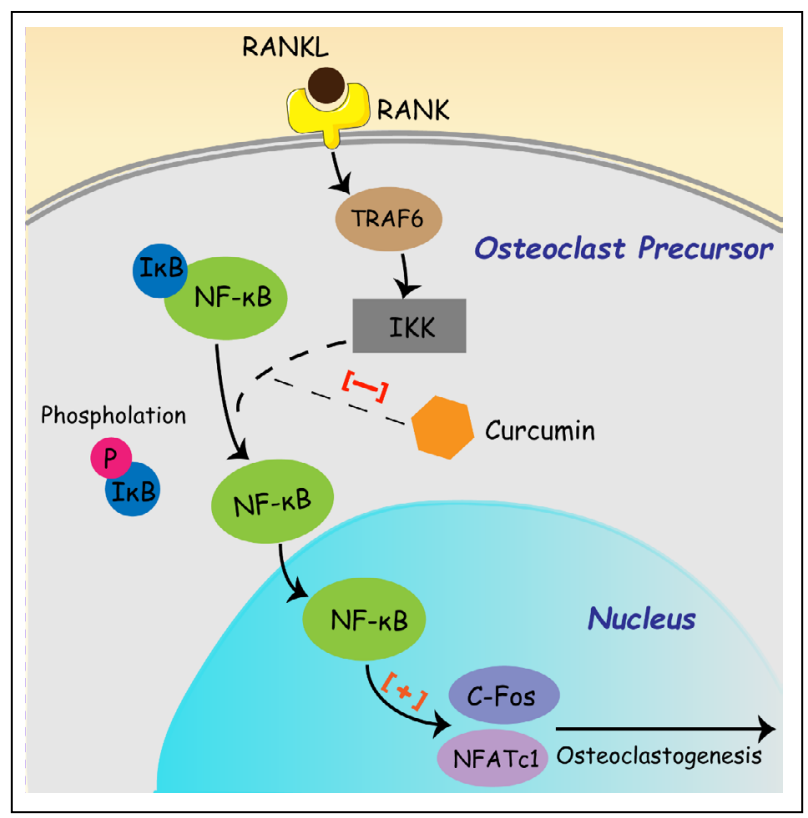

genes were expressed at lower levels in the curcumin groups, indicating downregulation in comparison with the PE group $(\mathrm{P}<0.01)$.

Expression of I $\kappa \mathrm{B} \alpha, \mathrm{P}-\mathrm{I} \kappa \mathrm{B} \alpha, \mathrm{c}-\mathrm{Fos}$, and NFATc1 proteins was then detected by Western blotting in cell samples from the blank, PE, low-dose curcumin, and high-dose curcumin groups (Fig. 4a, 4c) and in tissue samples from the blank, PE, solvent, and curcumin groups (Fig. 4b, 4d). The results of statistical analysis are shown in Fig. 4e and 4f. Levels of the factors involved in osteoclastogenesis, namely, c-Fos and NFATc1, were consistently increased in the cell lines and tissue samples treated with PE particles when compared with the corresponding blank groups (Fig. 4c, $4 \mathrm{~d}$; $\mathrm{P}<0.01$ ) and could be attenuated significantly by curcumin $(\mathrm{P}<0.01)$. In the tissue samples, the c-Fos and NFATc1 expression levels in the solvent group were not significant different from those in the PE group, and were significantly higher than those in the curcumin group $(\mathrm{P}<0.05)$. In both experiments, I $\mathrm{B} \alpha \alpha$ showed the lowest expression in the PE groups $(\mathrm{P}<0.05)$. The expression levels of $\mathrm{I} \kappa \mathrm{B} \alpha$ protein that were decreased by PE were attenuated in the low-dose and high-dose curcumin groups (cell samples, $\mathrm{P}<0.05$; tissue samples, $\mathrm{P}<0.01$ ). Interestingly, when compared with the decreased levels of I $\mathrm{KB} \alpha$ protein in the PE group, there was a significant increase in phosphorylated $\mathrm{I} \kappa \mathrm{B} \alpha$ levels in both the cell and tissue samples (Fig. $4 \mathrm{a}, 4 \mathrm{~b} ; \mathrm{P}<0.01$ ) that could be attenuated by curcumin $(\mathrm{P}<0.01)$. The $\mathrm{P}-\mathrm{I} \kappa \mathrm{B} \alpha$ protein levels in the solvent group were markedly higher than those in the curcumin group $(\mathrm{P}<0.05)$ but were also lower than those in the PE group.

Our present findings, combined with those of other researchers where RANK, c-Fos, and NFATc1 were found to be involved in osteoclastogenesis $[6-8,30]$ suggest that PE-induced osteoclastogenesis may occur via activation of a RANK/c-Fos/NFATc1 signaling pathway. Curcumin might attenuate PE-induced osteoclastogenesis by inhibiting this signaling pathway. These mechanism findings were simply presented in the schematic drawing in Fig. 5.

\section{Discussion}

PE particles produced by an artificial joint have been increasingly recognized as having the potential to induce inflammation and osteolysis [31-33], which would directly result in failure of artificial joint replacement. In this study, we confirmed that $1 \mathrm{mg} / \mathrm{mL}$ or $10 \mathrm{mg}$ of PE particles could trigger differentiation of osteoclasts in a RAW264.7 (monocyte/macrophage) 


\section{Cellular Physiology Cell Physiol Biochem 2018;50:1100-1112

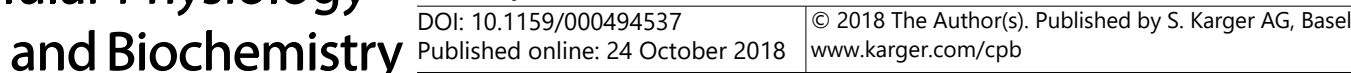

An et al.: Curcumin Inhibits Osteolysis Via Repressing NF-kB

cell line in vitro and in a murine calvaria model in vivo using TRAP straining and micro-CT scanning (Figs. 1 and 2, respectively). The murine calvaria model had been proven to be a successful and effective way of inducing osteogenesis [24,34] and TRAP staining is believed to be a specific detection method for osteoclasts [17]. Nevertheless, the mechanisms of wear debris (including PE)-induced inflammation and osteolysis remain a mystery [35].

NF-kB and its ligand, RANK, have been found to play a critical role in wear debrisinduced inflammation and osteoclastogenesis [36]. Activation of the NF- $\kappa B$ signaling pathway is essential for proliferation, differentiation, and maturation of osteoclasts [37]. After RANK binds with the RANK ligand, IKK is activated and subsequently phosphorylates

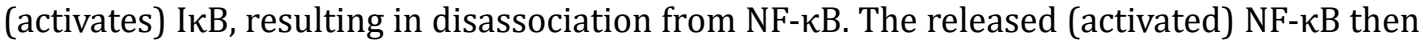
transfers into nuclei [38], where it increases expression levels of c-Fos and NFATc1, both of which are key factors in osteoclastogenesis [39]. Zhao et al. [40] found that aesculin could attenuate activation of NF- $\kappa B$ upon induction of RANKL, leading to decreased NFATc1 mRNA expression. With this mechanism in mind, we hypothesized that detecting the amount of mRNA in c-Fos and NFATc1 as well as the activity of P-IкB combined with histomorphologic examination could provide indirect evidence of the osteoclastogenic activity of PE and the mechanism of the anti-osteoclastogenic effect of curcumin.

To further explore whether PE induces differentiation of osteoclasts via the RANK/NF$\mathrm{kB}$ signaling pathway, we tested the expression and activation of the c-Fos, NFATc1, and IкB $\alpha$ proteins and found that c-Fos and NFATc1 were upregulated and $\mathrm{I} \kappa \mathrm{B} \alpha$ was activated by PE to trigger cell proliferation/differentiation in both a RAW264.7 cell line in vitro and a mouse model of calvarial bone in vivo (Figs. 3 and 4). In a study by Granchi et al. [41], trabecular bone cells were exposed to wear debris-like alumina or PE particles and it was found that differentiation of osteoclasts occurred in response to upregulation of related genes, namely, c-fms, RANK, c-src, c-Fos, TRAP, and calcitonin receptor. Combining these with results in similar systems where PE-induced differentiation of osteoclasts, these findings suggest that PE induces formation of osteoclasts via a RANK/NF-IкB/c-Fos cell proliferation/ differentiation signaling pathway.

Previous researchers have identified that curcumin has a significant ability to suppress the NF- $\kappa B$ signaling pathway [42]. Zhang et al. [43] verified that curcumin could inhibit activation of NF- $\mathrm{KB}$, which has an important role in the inflammatory response, in the lungs of rats with diabetes, thereby alleviating experimentally induced lung inflammatory injury. In a study of peripheral blood mononuclear cells from patients with rheumatoid arthritis, Wei et al. [44] found that curcumin could inhibit the osteoclastogenic potential of these cells via suppression of the MAPK/RANK/c-Fos/NFATc1 signaling pathway. Curcumin has also been confirmed to affect the process of osteoclastogenesis associated with metastatic breast cancer or periodontitis [34, 45], but an anti-osteoclastogenic effect of curcumin on periprosthetic osteolysis after THA has not yet been demonstrated. In our model of PEinduced osteoclastogenesis, we clearly demonstrated that curcumin attenuated PE-induced differentiation of osteoclasts in RAW264.7 cells in vitro and in a murine calvarial bone model in vivo by TRAP staining and micro-CT scanning. These results add to the evidence for the anti-osteoclastogenic activity of curcumin.

We then proceeded to molecular biology experiments to explore the specific mechanism of this intervention. Real-time PCR results revealed that the PE group had the highest level of c-Fos and NFATc1 mRNA. c-Fos and NFATc1 have been shown to play a critical role in initiating the process of osteolysis, so upregulation of these two factors indirectly suggests that they could trigger the osteolysis process. Combined with the results of TRAP staining and micro-CT scanning, our findings indicate that this signaling is involved in PE-induced osteolysis. These data show that curcumin exerted an inhibitory effect by downregulating the expression of the c-Fos and NFATc1 genes.

The osteolysis induced by PE has been confirmed previously by TRAP staining and microCT scanning. In addition, curcumin has already been reported to inhibit IKK [46], which phosphorylates $I \kappa B \alpha$ and helps $N F \kappa B$ to translocate into the nuclei of cells where it triggers cellular proliferation and differentiation. Therefore, analysis of $\mathrm{IkB} \alpha$ activity could provide 
critical information regarding the status of $\mathrm{IKK} / \mathrm{IkB} \alpha / \mathrm{NFkB}$ signaling and may indirectly indicate its involvement in osteolysis. For this reason, we detected the activity of P-IkB $\alpha$ by Western blot in addition to expression levels of c-Fos and NFATc1 protein. The difference in expression of c-Fos and NFATc1 protein was almost in accordance with our results for gene expression except that there was no significant difference between the low-dose and highdose curcumin groups, suggesting that a very small amount of curcumin could markedly inhibit expression of these factors (Fig. 4e, 4f). In the in vitro (RAW264.7 cell) samples, we found that the PE group had the lowest IkB $\alpha$ protein level but the highest P-IkB $\alpha$ activity and that this activity could be inhibited by curcumin (Fig. 4e). Results similar to the in vitro data were obtained for the in vivo samples (murine calvaria); that is, the PE group had a lower $\mathrm{IkB} \alpha$ protein level but had the highest activity, and curcumin could prevent this PE-induced activity (Fig. 4f). These findings indicate that the mechanism of the anti-osteolysis activity of curcumin may be via inhibition of IKK resulting in dephosphorylation of IkB $\alpha$ which binds to NFkB to prevent its nuclear translocation and thereby inhibits the osteolytic process.

In the in vivo experiments, the solvent (DMSO and corn oil) group showed a limited ability to increase c-Fos and NFATc1 expression and dephosphorylated IkB levels when compared with the blank group. However, the curcumin dissolved in the solvent had a greater ability to prevent PE-induced expression of the c-Fos and NFATc1 genes $(\mathrm{P}<0.05)$ and attenuated PE-induced I $\kappa B$ phosphorylation $(\mathrm{P}<0.05)$, so the anti-osteoclastogenic activity of curcumin was not impaired.

\section{Conclusion}

In conclusion, in this study we successfully established PE-induced osteoclast differentiation models in vitro (RAW246.7 cells) and in vivo (murine calvaria) that were verified by TRAP staining and micro-CT scanning. We subsequently found that curcumin could prevent PE-induced differentiation of osteoclasts in these models. Moreover, we verified that curcumin could inhibit c-Fos and NFATc1 genes and protein expression as well as IkBa activity, which may be the mechanism for the anti-osteolysis potential of curcumin. These data provide experimental and theoretical evidence that supports the use of curcumin as a treatment for osteolysis induced by wear particles.

\section{Acknowledgements}

This basic research was performed with the support of the Orthopedics Department and Institute of Medical Sciences of Xiangya Hospital. This manuscript has been edited and proofread by Medjaden Bioscience Limited. This study was supported by two National Natural Science Foundation of China (NSFC) projects (grant numbers: 81672138 and 81601883) and supported by the Fundamental Research Funds for the Central Universities of Central South University (grant numbers: 2016zzts1290 and 2018zzts257), and also supported by Hunan Provincial Natural Science Foundation of China (grant number: 2018JJ3861).

\section{Disclosure Statement}

The authors declare that they have no conflicts of interest pertaining to this study.

\section{References}

1 Cai P, Hu Y, Xie J: Large-diameter Delta ceramic-on-ceramic versus common-sized ceramic-on-polyethylene bearings in THA. Orthopedics 2012;35:e1307-1313. 


\section{Cellular Physiology Cell Physiol Biochem 2018;50:1100-1112 and Biochemistry \begin{tabular}{l|l} 
DOI: 10.1159/000494537 & $\begin{array}{l}\text { O } 2018 \text { The Author(s). Published by S. Karger AG, Basel } \\
\text { www.karger.com/cpb }\end{array}$
\end{tabular}

- Kurtz SM, Ong KL, Schmier J, Zhao K, Mowat F, Lau E: Primary and revision arthroplasty surgery caseloads in the United States from 1990 to 2004. J Arthroplasty 2009;24:195-203.

- Park KJ, Menendez ME, Barnes CL: Perioperative Periprosthetic Fractures Associated With Primary Total Hip Arthroplasty. J Arthroplasty 2016;10.1016/j.arth.2016.08.034.

-4 Goodship AE, Blunn GW, Green J, Coathup MJ: Prevention of strain-related osteopenia in aseptic loosening of hip prostheses using perioperative bisphosphonate. J Orthop Res 2008;26:693-703.

5 Wang L, Dai Z, Xie J, Liao H, Lv C, Hu Y: Alteration of the RANKL/RANK/OPG System in Periprosthetic Osteolysis with Septic Loosening. Inflammation 2016;39:218-227.

-6 Lacey DL, Timms E, Tan HL, Kelley MJ, Dunstan CR, Burgess T, Elliott R, Colombero A, Elliott G, Scully S, Hsu H, Sullivan J, Hawkins N, Davy E, Capparelli C, Eli A, Qian YX, Kaufman S, Sarosi I, Shalhoub V et al.: Osteoprotegerin ligand is a cytokine that regulates osteoclast differentiation and activation. Cell 1998;93:165-176.

-7 Jung SM, Kim KW, Yang CW, Park SH, Ju JH: Cytokine-mediated bone destruction in rheumatoid arthritis. J Immunol Res 2014;2014:263625.

8 Gudena R, Kuna S, Pradhan N: Aseptic loosening of total hip replacement presenting as an anterior thigh mass. Musculoskelet Surg 2013;97:247-249.

-9 Wilken R, Veena MS, Wang MB, Srivatsan ES: Curcumin: A review of anti-cancer properties and therapeutic activity in head and neck squamous cell carcinoma. Mol Cancer 2011;10:12.

10 Yang H, Xu W, Zhou Z, Liu J, Li X, Chen L, Weng J, Yu Z: Curcumin attenuates urinary excretion of albumin in type II diabetic patients with enhancing nuclear factor erythroid-derived 2-like 2 (Nrf2) system and repressing inflammatory signaling efficacies. Exp Clin Endocrinol Diabetes 2015;123:360-367.

11 Boyanapalli SS, Tony Kong AN: “Curcumin, the King of Spices": Epigenetic Regulatory Mechanisms in the Prevention of Cancer, Neurological, and Inflammatory Diseases. Curr Pharmacol Rep 2015;1:129-139.

$\checkmark 12$ Chin KY: The spice for joint inflammation: anti-inflammatory role of curcumin in treating osteoarthritis. Drug Des Devel Ther 2016;10:3029-3042.

13 Klinger NV, Mittal S: Therapeutic Potential of Curcumin for the Treatment of Brain Tumors. Oxid Med Cell Longev 2016;2016:9324085.

-14 Kunwar A, Priyadarsini KI: Curcumin and Its Role in Chronic Diseases. Adv Exp Med Biol 2016;928:1-25.

15 Li B, Hu Y, Zhao Y, Cheng M, Qin H, Cheng T, Wang Q, Peng X, Zhang X: Curcumin Attenuates Titanium Particle-Induced Inflammation by Regulating Macrophage Polarization In vitro and In vivo. Front Immunol 2017;8:55.

16 Simonet WS, Lacey DL, Dunstan CR, Kelley M, Chang MS, Luthy R, Nguyen HQ, Wooden S, Bennett L, Boone T, Shimamoto G, DeRose M, Elliott R, Colombero A, Tan HL, Trail G, Sullivan J, Davy E, Bucay N, RenshawGegg L et al.: Osteoprotegerin: a novel secreted protein involved in the regulation of bone density. Cell 1997;89:309-319.

17 Jules J, Wang S, Shi Z, Liu J, Wei S, Feng X: The IVVY Motif and Tumor Necrosis Factor Receptor-associated Factor (TRAF) Sites in the Cytoplasmic Domain of the Receptor Activator of Nuclear Factor kappaB (RANK) Cooperate to Induce Osteoclastogenesis. J Biol Chem 2015;290:23738-23750.

18 Kong X, Yang Y, Wu W, Wan H, Li X, Zhong M, Su X, Jia S, Lin N: Triterpenoid Saponin W3 from Anemone flaccida Suppresses Osteoclast Differentiation through Inhibiting Activation of MAPKs and NF-kappaB Pathways. Int J Biol Sci 2015;11:1204-1214.

19 Zhou F, Shen Y, Liu B, Chen X, Wan L, Peng D: Gastrodin inhibits osteoclastogenesis via down-regulating the NFATc1 signaling pathway and stimulates osseointegration in vitro. Biochem Biophys Res Commun 2017;484:820-826.

-20 von Metzler I, Krebbel H, Kuckelkorn U, Heider U, Jakob C, Kaiser M, Fleissner C, Terpos E, Sezer O: Curcumin diminishes human osteoclastogenesis by inhibition of the signalosome-associated I kappaB kinase. J Cancer Res Clin Oncol 2009;135:173-179.

-21 Boyce BF, Yamashita T, Yao Z, Zhang Q Li F, Xing L: Roles for NF-kappaB and c-Fos in osteoclasts. J Bone Miner Metab 2005;23 Suppl:11-15.

-22 Yang Y, Zhou H, Yang Y, Li W, Zhou M, Zeng Z, Xiong W, Wu M, Huang H, Zhou Y, Peng C, Huang C, Li X, Li G: Lipopolysaccharide (LPS) regulates TLR4 signal transduction in nasopharynx epithelial cell line 5-8F via NFkappaB and MAPKs signaling pathways. Mol Immunol 2007;44:984-992.

-23 Guo LJ, Liao L, Yang L, Li Y, Jiang TJ: MiR-125a TNF receptor-associated factor 6 to inhibit osteoclastogenesis. Exp Cell Res 2014;321:142-152. 


\section{Cellular Physiology Cell Physiol Biochem 2018;50:1100-1112

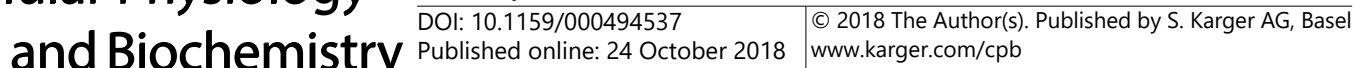

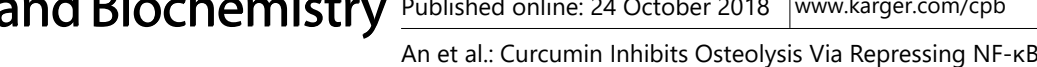

-24 Bharti AC, Takada Y, Aggarwal BB: Curcumin (diferuloylmethane) inhibits receptor activator of NF-kappa B ligand-induced NF-kappa B activation in osteoclast precursors and suppresses osteoclastogenesis. J Immunol 2004;172:5940-5947.

25 HAN F HY, AN S, LEI P, WANG L: In vivo experiment of curcumin treating polyethylene particle induced osteolysis. Journal of Clinical and Pathological Research 2017;37:331-338.

-26 Zimmermann M: Ethical guidelines for investigations of experimental pain in conscious animals. Pain 1983;16:109-110.

-27 Panmekiate S, Ngonphloy N, Charoenkarn T, Faruangsaeng T, Pauwels R: Comparison of mandibular bone microarchitecture between micro-CT and CBCT images. Dentomaxillofac Radiol 2015;44:20140322.

-28 Huynh NC, Everts V, Nifuji A, Pavasant P, Ampornaramveth RS: Histone deacetylase inhibition enhances invivo bone regeneration induced by human periodontal ligament cells. Bone 2016;95:76-84.

29 Burstone MS: Histochemical demonstration of acid phosphatase activity in osteoclasts. J Histochem Cytochem 1959;7:39-41.

30 Ingham E, Fisher J: Biological reactions to wear debris in total joint replacement. Proc Inst Mech Eng H 2000;214:21-37.

31 Zawawi MS, Marino V, Perilli E, Cantley MD, Xu J, Purdue PE, Dharmapatni AA, Haynes DR, Crotti TN: Parthenolide reduces empty lacunae and osteoclastic bone surface resorption induced by polyethylene particles in a murine calvarial model of peri-implant osteolysis. J Biomed Mater Res A 2015;103:35723579.

-32 Nabeshima A, Pajarinen J, Lin TH, Jiang X, Gibon E, Cordova LA, Loi F, Lu L, Jamsen E, Egashira K, Yang F, Yao Z, Goodman SB: Mutant CCL2 protein coating mitigates wear particle-induced bone loss in a murine continuous polyethylene infusion model. Biomaterials 2017;117:1-9.

-33 Ormsby RT, Cantley M, Kogawa M, Solomon LB, Haynes DR, Findlay DM, Atkins GJ: Evidence that osteocyte perilacunar remodelling contributes to polyethylene wear particle induced osteolysis. Acta Biomater 2016;33:242-251.

34 Zhou T, Chen D, Li Q Sun X, Song Y, Wang C: Curcumin inhibits inflammatory response and bone loss during experimental periodontitis in rats. Acta Odontol Scand 2013;71:349-356.

35 Goodman SB: Wear particles, periprosthetic osteolysis and the immune system. Biomaterials 2007;28:5044-5048.

36 Abu-Amer Y: NF-kappaB signaling and bone resorption. Osteoporos Int 2013;24:2377-2386.

37 Jiang J, Jia T, Gong W, Ning B, Wooley PH, Yang SY: Macrophage Polarization in IL-10 Treatment of ParticleInduced Inflammation and Osteolysis. Am J Pathol 2016;186:57-66.

38 Karin M: How NF-kappaB is activated: the role of the IkappaB kinase (IKK) complex. Oncogene 1999;18:6867-6874.

39 Li Y, Zhong G, Sun W, Zhao C, Zhang P, Song J, Zhao D, Jin X, Li Q, Ling S, Li Y: CD44 deficiency inhibits unloading-induced cortical bone loss through downregulation of osteoclast activity. Sci Rep 2015;5:16124.

$\$ 40$ Zhao XL, Chen LF, Wang Z: Aesculin modulates bone metabolism by suppressing receptor activator of NF-kappaB ligand (RANKL)-induced osteoclastogenesis and transduction signals. Biochem Biophys Res Commun 2017;488:15-21.

-41 Granchi D, Amato I, Battistelli L, Ciapetti G, Pagani S, Avnet S, Baldini N, Giunti A: Molecular basis of osteoclastogenesis induced by osteoblasts exposed to wear particles. Biomaterials 2005;26:2371-2379.

42 Xu F, Lin SH, Yang YZ, Guo R, Cao J, Liu Q: The effect of curcumin on sepsis-induced acute lung injury in a rat model through the inhibition of the TGF-beta1/SMAD3 pathway. Int Immunopharmacol 2013;16:1-6.

-43 Zhang F, Yang F, Zhao H, An Y: Curcumin alleviates lung injury in diabetic rats by inhibiting NF-kappaB pathway. Clin Exp Pharmacol Physiol 2015;10.1111/1440-1681.12438.

44 Shang W, Zhao LJ, Dong XL, Zhao ZM, Li J, Zhang BB, Cai H: Curcumin inhibits osteoclastogenic potential in PBMCs from rheumatoid arthritis patients via the suppression of MAPK/RANK/c-Fos/NFATc1 signaling pathways. Mol Med Rep 2016;14:3620-3626.

45 Thamake SI, Raut SL, Gryczynski Z, Ranjan AP, Vishwanatha JK: Alendronate coated poly-lactic-co-glycolic acid (PLGA) nanoparticles for active targeting of metastatic breast cancer. Biomaterials 2012;33:71647173.

46 Xu H, Cheng L, Wang C, Ma X, Li Y, Liu Z: Polymer encapsulated upconversion nanoparticle/iron oxide nanocomposites for multimodal imaging and magnetic targeted drug delivery. Biomaterials 2011;32:93649373. 